\title{
3D Printing for Fast Prototyping of Pharmaceutical Dissolution Testing Equipment for Nonstandard Applications
}

\author{
Przemysław Dorożyński ${ }^{1 *}$, Witold Jamróz ${ }^{2}$, Władysław P. Węglarz ${ }^{3}$, Wojciech Kulinowski ${ }^{4}$, Mateusz \\ Zaborowski ${ }^{2}$, and Piotr Kulinowski ${ }^{4}$ \\ ${ }^{1}$ Pharmaceutical Research Institute, Warszawa, Poland \\ ${ }^{2}$ Department of Pharmaceutical Technology and Biopharmaceutics, Jagiellonian University Medical College, Kraków, Poland \\ ${ }^{3}$ Department of Magnetic Resonance Imaging, Institute of Nuclear Physics, Polish Academy of Sciences, Kraków, Poland \\ ${ }^{4}$ Department of Mathematics, Physics and Technical Science, Institute of Technology, Pedagogical University of Cracow, Kraków, Poland
}

e-mail:mfdorozy@cyf-kr.edu.pl

\section{ABSTRACT}

Purpose of the research was to assess feasibility of fused deposition techniques (3D printing) for development of analytical equipment dedicated for specific dosage forms and for nonstandard applications. Dissolution profiles as well as 3D magnetic resonance imaging (MRI) of the buccal tablets during hydration in dissolution medium were analyzed. The principal result of the study was the first working example of rapid 3D prototyping of dedicated, MRI-compatible dissolution equipment for mucoadhesive buccal tablets. Rapid prototyping techniques were found to be a fast, inexpensive way to develop a dedicated dissolution testing setup.

KEYWORDS: additive manufacturing, 3D printing, solid free-form fabrication, buccal bioadhesive tablets, 3D ultrashort echo time magnetic resonance imaging (3D UTE MRI), pharmaceutical dissolution testing equipment

\section{INTRODUCTION}

( he number of studies concerning application of three dimensional (3D) printing techniques in pharmaceutical technology has grown continuously since 2005, but the main interest in application of these techniques is focused on the manufacturing of new customized dosage forms (1). As a successful example of applying this technology, the first 3D-printed drug product containing leviteracetam, Spritam, was approved by U.S. Food and Drug Administration (FDA) in 2015 (2).

Although the direct manufacturing of dosage forms using 3D technology is a fascinating topic, it does not exhaust the possibilities offered by 3D printing for pharmaceutical technology. Among possible options, rapid prototyping and rapid tooling should be taken under consideration. When using traditional methods for preparation of 3D objects, it is necessary to remove excess material by machining or forming objects with a casting technique. Both of these techniques require the involvement of highly specialized equipment such as drills, milling machines, grinders, or usage of high temperatures during injection molding of the materials to the appropriate forms, which prevents their use in random manufacturing sites. The 3D printing technology is devoid of such disadvantages.

The important area where the rapid prototyping and tooling may find a wide range of applications is the development of analytical equipment dedicated for specific dosage forms and for nonstandard applications. The variety of novel drug delivery systems (e.g., orodispersible tablets and films, intraoral mucoadhesive tablets, etc.) generates serious problems with the relevant dissolution testing $(3,4)$. Moreover, some specific testing methods of drug delivery systems require dedicated tools (e.g., holders) (5).

Dissolution studies belong to the most frequently applied biopharmaceutical tests for the evaluation of solid dosage forms. The pharmacopoeial methodology of dissolution testing, although well established, is often insufficient for evaluation of new drug delivery systems. Despite this fact, the possibility of development of instrumentation dedicated for specific drug delivery systems with 3D printing is practically not explored. The first and only attempt of application of fused deposition modeling as

*Corresponding author 
a quick prototyping method of dedicated equipment for monitoring of tablet hydration was presented by Charest et al. (6). The authors produced tablet holders using a fused deposition method (FDM). These holders are suitable to carry out standard dissolution studies as well as magnetic resonance imaging (MRI) of the dissolving tablets.

Drug release in vitro is a prerequisite for drug absorption studies in vivo, and for this purpose, the bioavailability of the drug is important (7). In vitro drug dissolution studies provide rich information about the properties of tested dosage forms but lack of specificity. Despite the lack of specificity, the dissolution test is sensitive to physicochemical changes in the drug product and is widely used for evaluation of drug formulations. The combination of dissolution testing with imaging techniques, such as MRI or Fourier transform infrared (FTIR) microscopy, gives the possibility of spatiotemporal characterization of dosage forms during hydration and drug release and consequently can increase specificity (8-10).

The aim of our work was to present the possibility of additive manufacturing (3D printing technique) for fast prototyping of elements of dissolution equipment for rare or nonstandard drug delivery systems. As a working example, we developed instrumentation for a combined dissolution test and MRI study of intraoral mucoadhesive tablets.

\section{MATERIALS AND METHODS \\ Pharmaceutical Materials}

In order to prepare mucoadhesive tablets Bromamine $\mathrm{T}$ (BAT, sodium N-bromo-p-toluene sulfonamide) synthesized by the Insbruck Medical University (Department of Hygiene, Microbiology, and Social Medicine, Division of Hygiene and Medical Microbiology), Carbopol 940 (Caesar \& Loretz GmbH, Hilden, Germany), Neusilin US2 (Fuji Chemical Industries Co., Ltd., Japan), Fujicalin (Fuji Chemical Industries Co., Ltd., Japan), Aeroperl 300 (Evonik Industries, Essen, Germany) were used. All other materials were of reagent grade and used without further purification.

\section{Preparation of Model Bioadhesive Tablets}

Model bioadhesive flat-faced bevel-edge tablets, $6 \mathrm{~mm}$ in diameter, were prepared by direct compression in a Korsch EKO eccentric tablet press (Korsch AG, Berlin, Germany) equipped with a mutli-tip punch and die. The tablets were composed of $20 \%$ BAT and $40 \%$ Carbopol. Before tableting, the active substance and excipients were mixed manually in mortar.

\section{Designing and Printing the Insert}

The first phase of preparing a 3D-printed insert for buccal tablets was calculating the dimensions of the inner part of the flow-through cell and preparing a technical drawing (Fig. 1a), which was the basis of 3D model. The 3D model was designed using free and open-source software (Blender, version 2.73a, www.blender.org). The 3D model was created by modification of the cylinder mesh. Major tools used during this process were loop operations, face and vertex removals, solidification, boolean modifiers, as well as face extrusion. Before printing, the evaluation of 3D model mesh was performed by a Blender internal module, and the absence of non-manifold edges and bad contiguous edges was verified. The 3D object was exported as a StereoLithography (STL) file format.

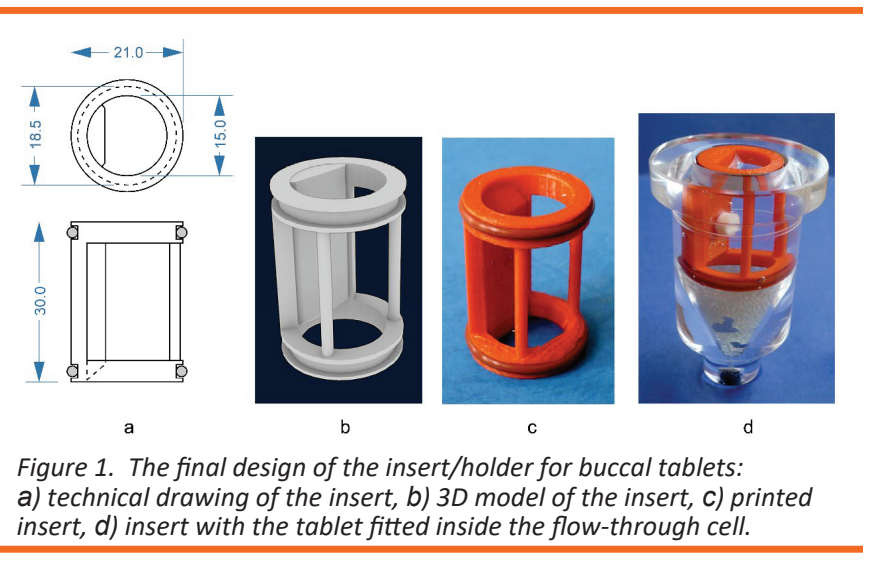

The 3D printing was carried out with 3DKreator Motion (3DKreator, Kraków, Poland). Thermoplastic acrylonitrile butadiene styrene filament was used as building material. Prototypes of the flow-through cell inserts were printed and evaluated. After introducing some design modifications, the prototype was printed again.

\section{Dissolution Testing}

The dissolution study was carried out using USP apparatus 4 (DFZ with piston pump HPK 60, Erweka, Heusenstamm, Germany). The apparatus was equipped with a pharmacopoeial 22.6-mm flow-through cell for tablets and capsules. The tablets were mounted to the 3D-printed insert by cyanoacrylic glue, and the insert was fitted in the flow-through cell by two silicon O-rings (Fig 1).

The dissolution medium, phosphate buffer $(\mathrm{pH}$ 6.8) was circulated in the closed loop with the minimum piston pump circulation rate $(4.5 \mathrm{~mL} / \mathrm{min})$. The temperature in the dissolution cell was maintained at $37 \pm 0.5{ }^{\circ} \mathrm{C}$. The samples of dissolution media were withdrawn after first $30 \mathrm{~min}$ and every hour up to 5 hours. The volume of the 
samples after 30 and $60 \mathrm{~min}$ was $10 \mathrm{~mL}$, and the volume of the subsequent samples was $20 \mathrm{~mL}$. Equal amounts of dissolution media were replaced immediately after withdrawal of the medium sample. The amounts of released BAT were determined by measuring the UV absorption at $224 \mathrm{~nm}$ and calculated using a calibration curve of the drug. Drug release tests were conducted in triplicate and the results are reported as the mean \pm SD.

\section{D MRI}

Tablets were mounted in the holder, the same way as in dissolution studies. The holder was put into the polycarbonate chamber filled with $30 \mathrm{~mL}$ of dissolution medium and placed in a 9.4 T research tomograph (Bruker BioSpin MRI GmbH, Ettlingen, Germany). The studies were carried out in room temperature $\left(\sim 25{ }^{\circ} \mathrm{C}\right)$. The images were taken after 1, 2, 3, and 4 hours using the 3D ultra-short echo time (UTE) MR pulse imaging sequence. The following parameters of the pulse sequence were applied: number of scans $=1$, flip angle $=5^{\circ}$, echo-time $=$ $20 \mu \mathrm{s}$, repetition time $=8 \mathrm{~ms}$, slice thickness $=26.3 \mathrm{~mm}$, field of view $=2.63 \mathrm{~cm}^{3}$, image matrix size $=256 \times 256 \times$ 256 pixels.

Image segmentation was performed using a Trainable Weka Segmentation (TWS) plug-in module (http://fiji.sc/ Trainable_Weka_Segmentation) (11), part of the public domain software package, Fiji (https://fiji.sc) (12). The whole image volume was cropped to $185 \times 138 \times 124$ pixels, including a swollen tablet, to reduce image segmentation time. Four regions were assumed: dissolution medium, swollen fully hydrated, partially hydrated, and dry matrix. For other details of image segmentations procedure, see the work by Kulinowski et al. (10). Based on the results of image segmentations, volumes of the segmented regions of the matrix were calculated.

\section{RESULTS AND DISCUSSION}

Due to specific conditions occurring in the oral cavity, it is necessary to develop dosage forms that could be easily placed in the action site and subsequently removed after a specified amount of time. The mucoadhesive tablet may be placed on the buccal mucosa, which limits dissolution of the active substance from the adhered surface of the tablet. The amount of liquid for drug dissolution in the mouth is very low; the volume of saliva in healthy volunteers ranges from $0.66 \mathrm{~mL}$ in females to $0.87 \mathrm{~mL}$ in males, with a flow-rate of $0.1-0.3 \mathrm{~mL} / \mathrm{min}$ in unstimulated state and increases to $0.5-1.5 \mathrm{~mL} / \mathrm{min}$ during stimulated flow (13). Despite this, most in vitro dissolution studies concerning buccal drug delivery systems are typically carried out using USP Apparatus 1 or 2 in various media volumes, ranging from 300 to $400 \mathrm{~mL}(14,15)$.

In contrast to previously published studies, experimental conditions in the present study were arranged as an approach to biorelevant dissolution of buccal tablets. For this purpose, the insert/tablet holder was designed to allow hydration of the buccal bioadhesive dosage form from one side and to be applied with standard apparatus 4 equipment. The 3D-printing technology gave us the opportunity for verification of project assumptions and for introduction of design modifications. The final design of the insert is presented in Figure 1. The dimensions of the insert were adjusted to allow placement in the 22.6- $\mathrm{mm}$ flow-through cell. The height of the insert was $30.0 \mathrm{~mm}$ and its diameter was $21.0 \mathrm{~mm}$. The insert was positioned within the flow-through cell by two O-rings.

The tablets were mounted to the insert using cyanoacrylate adhesive. The mounting of the tablets on the back wall of the insert reduced their active dissolution surface, mimicking the application in the oral cavity. Dissolution data are presented in Table 1, and the drug dissolution profile is presented in Figure 2. After 4 hours, $50.2 \%$ of BAT was released from the tablets, and no burst effects were observed.

Table 1. Juxtaposition of Dissolution and MRI Results

\begin{tabular}{|c|c|c|c|c|c|}
\hline \multirow[b]{2}{*}{ Time (h) } & \multirow{2}{*}{$\begin{array}{c}\text { BAT } \\
\text { dissolution } \\
\text { (\%) }\end{array}$} & \multicolumn{4}{|c|}{ Region volume $\left(\mathrm{mm}^{3}\right)$} \\
\hline & & Total & Dry & $\begin{array}{l}\text { Partially } \\
\text { hydrated }\end{array}$ & $\begin{array}{c}\text { Fully } \\
\text { hydrated }\end{array}$ \\
\hline 0 & 0 & 76 & 76 & 0 & 0 \\
\hline 1 & 14.9 & 286 & 32 & 21 & 232 \\
\hline 2 & 26.2 & 289 & 23 & 26 & 240 \\
\hline 3 & 39.6 & 295 & 23 & 14 & 257 \\
\hline 4 & 50.2 & 425 & 23 & 1 & 401 \\
\hline
\end{tabular}

MRI, magnetic resonance imaging; BAT: Bromamine T.

The visual observation of the tablets during drug dissolution showed the changes in size, color, and transparency of the hydrated polymeric matrix (Fig. 3). After contact with the dissolution medium, the tablet swelled effectively. After $4 \mathrm{~h}$, the swollen hydrogel filled almost the whole dissolution chamber. The hydrogel that formed on the surface was white and opaque while the core changed its color to yellow; therefore, it was difficult to assess the internal structure of the swollen tablet.

MRI results are presented in Figures 4-6. Figure 4 presents orthoslice views of 3D images at four chosen time points. 
In the MR images, the swollen matrix is slightly brighter than the surrounding medium due to $T_{1}$-weighting caused by short repetition time of the imaging pulse sequence. The MRI results also revealed the regions of low intensity, indicating the volume of unhydrated and partially hydrated polymer, which could be the reservoir of undissolved BAT as well as air voids inside the swollen part of the matrix. Regarding pharmaceutical application of MRI methods, the 2D ultra-short echo time pulse sequence (UTE) has been previously applied for evaluation of enteric coated tablets (16). In the current study, 3D UTE MRI was carried out for the first time for evaluation of the pharmaceutical dosage forms (mucoadhesive tablets) in vitro.

Figure 5 presents rendered 3D images of segmented regions obtained from $3 \mathrm{D}$ image volume acquired at 1 hour. The box containing the swollen tablet is a 1.27 $\times 1.62 \times 0.77 \mathrm{~cm}^{3}(124 \times 158 \times 75$ pixels $)$ volume. Three separate volumes are presented corresponding to three regions (three hydration regimes) assumed for volume segmentation: dry matrix, partially hydrated, and fully hydrated. Due to extremely short echo time $(20 \mu \mathrm{s})$, the most internal volume could be denoted as a true dry volume. The dry/partially hydrated and partially/fully hydrated borders were relatively smooth. Consequently, the partially hydrated volume formed a regular, positive meniscus-like shape. The external border of the hydrated matrix volume was irregular (fuzzy) and asymmetric. The results are in agreement with data presented in Figure 4, and they offer much more in-depth information.

Temporal evolution of the segmented regions is presented in Table 1 and in Figure 6 by means of calculated volumes of the matrix. The data obtained allow assessment of matrix swelling. During the first hour of hydration, total volume of the tablet increased more than 3 times compared to volume of the dry tablet (from 76 to $286 \mathrm{~mm}^{3}$ ). Between the first and third hour of hydration, the volume was stable (slight increase was observed, up to $295 \mathrm{~mm}^{3}$ ). At the fourth hour, total volume of the matrix increased to $425 \mathrm{~mm}^{3}$ (more than 5.5 times increase compared to dry matrix).

During the entire dissolution time, the fully hydrated matrix constituted most of the matrix volume (from $81 \%$ of total matrix volume at $1 \mathrm{~h}$ to $94 \%$ at 4 hours of hydration). Dry part of the matrix reduced quickly from 76 to $32 \mathrm{~mm}^{3}$ during the first hour of hydration, then it stabilized at $23 \mathrm{~mm}^{3}$. It constituted $11 \%$ of total matrix volume at 1 hour, and it reduced to $5 \%$ at 4 hours. The partially hydrated region constituted less than $10 \%$ of the matrix volume and disappeared completely at 4 hours.

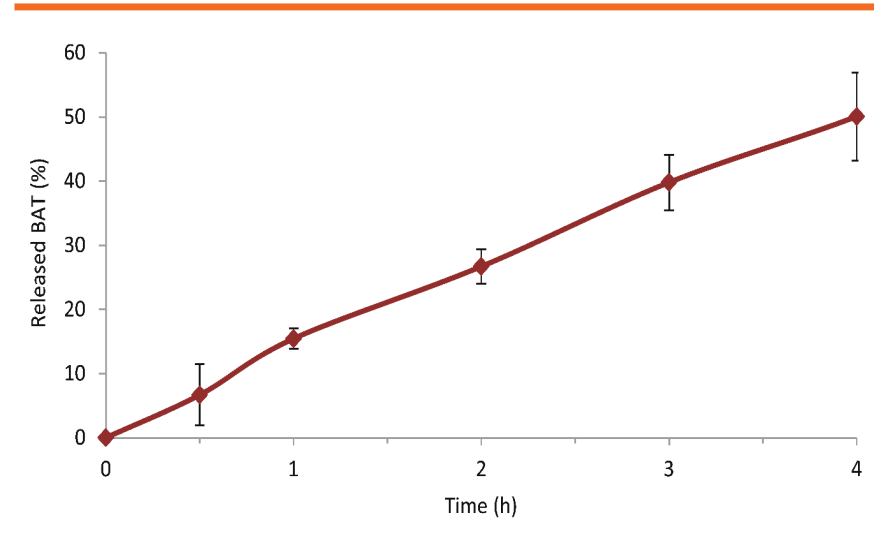

Figure 2. Dissolution profile of Bromamine $T(B A T)$ from bioadhesive tablets $(n=3)$.

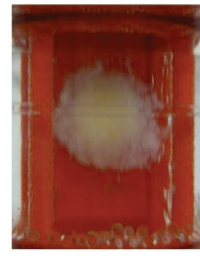

$1 \mathrm{hr}$

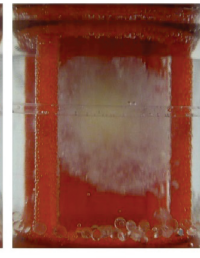

$2 \mathrm{hrs}$

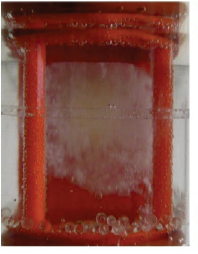

$3 \mathrm{hrs}$

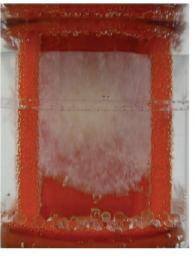

4 hrs
Figure 3. Swelling of the tablet within the insert during dissolution.

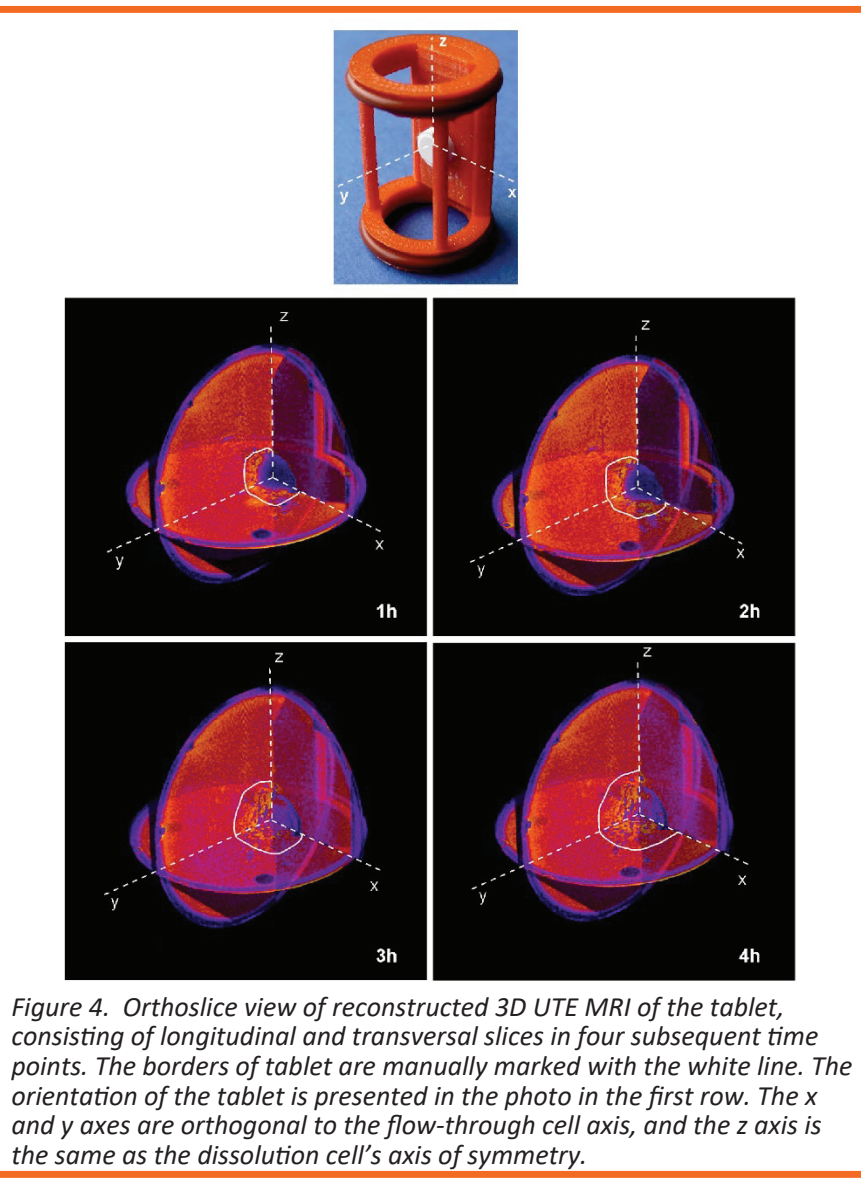


In this study, 3D imaging was chosen according to highly irregular, fuzzy-like, swelling of the buccal tablets. Previous MRI studies of matrix tablets have been performed using 2D imaging techniques due to regular swelling/hydration of the matrix; in these studies, central axial, coronal, and sagittal cross sections of the hydrated matrix have given unambiguous characteristics of the swelling system (17).
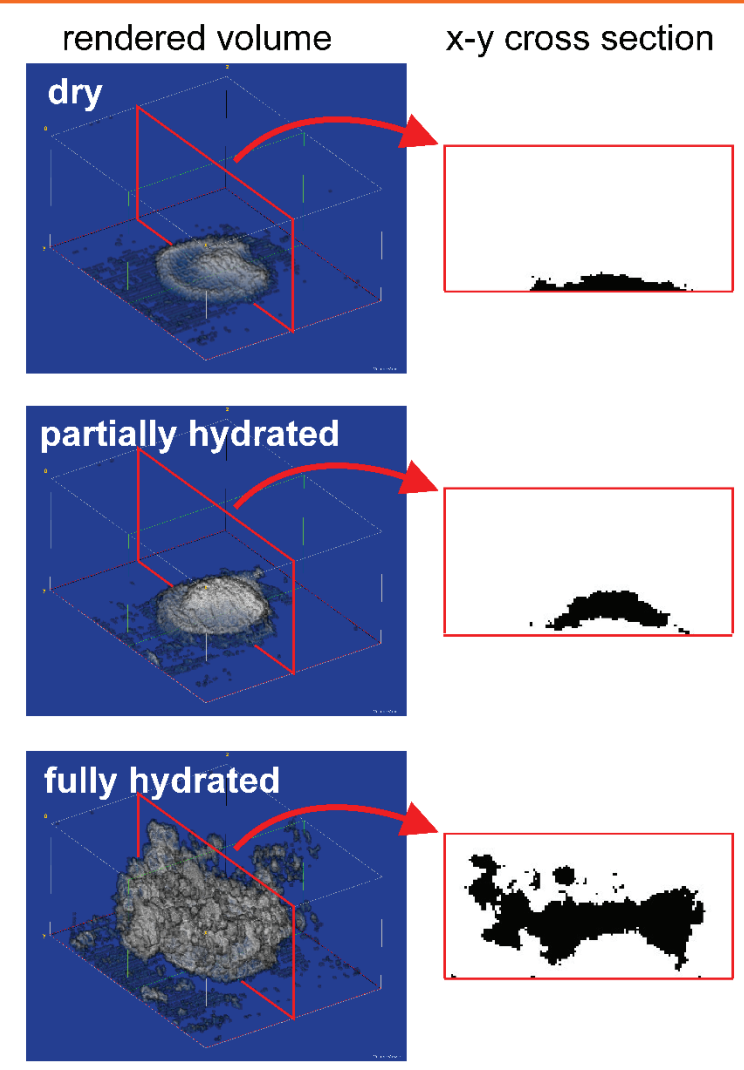

Figure 5. Rendered MRI volumes and $x-y$ axial cross sections of the regions (different hydration regimes) inside the swollen matrix tablet at 1 h of hydration.

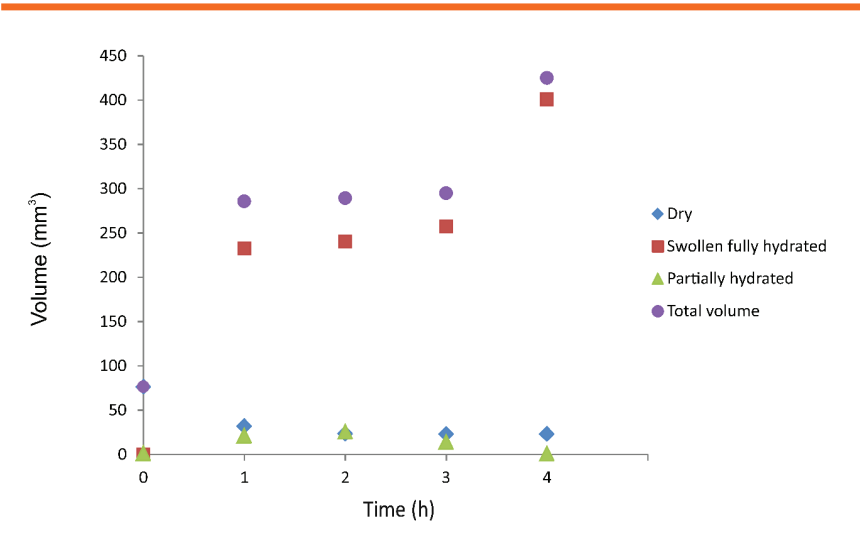

Figure 6. Temporal evolution of the particular regions/volumes of tablet as assessed by MRI and image segmentation techniques.

\section{CONCLUSIONS}

The work presents the first working example of rapid 3D prototyping applied to development of dedicated dissolution equipment, which we demonstrated using mucoadhesive buccal tablets that undergo hydration and swelling from one side only. Dissolution profiles, as well as 3D MR images of the buccal tablets during hydration in dissolution medium obtained using a 3D-printed insert/tablet holder were presented. This study showed that specific problems in dissolution testing setup development can be solved iteratively in a relatively short time using additive manufacturing.

\section{ACKNOWLDEGEMENTS}

The authors disclosed no funding related to this study.

\section{CONFLICT OF INTEREST}

The authors disclosed no conflicts of interest related to this article.

\section{REFERENCES}

1. Jonathan, G.; Karim, A. 3D printing in pharmaceutics: A new tool for designing customized drug delivery systems. Int. J. Pharm. 2016, 499, 376-394. DOI: 10.1016/j.ijpharm.2015.12.071.

2. Press Release: FDA Approves the First 3D Printed Drug Product. Aprecia Pharmaceuticals website. Published 2015. Accessed June 2, 2017. https://www.aprecia.com/pdf/2015_08_03_ Spritam_FDA_Approval_Press_Release.pdf.

3. Culen, M.; Rezacova, A.; Jampilek, J.; Dohnal, J. Designing a dynamic dissolution method: a review of instrumental options and corresponding physiology of stomach and small intestine. J. Pharm. Sci. 2013, 102, 2995-3017. DOI: 10.1002/jps.23494.

4. Garbacz, G.; Klein, S. Dissolution testing of oral modified-release dosage forms. J. Pharm. Pharmacol. 2012, 64, 944-968. DOI: 10.1111/j.2042-7158.2012.01477.x.

5. Dorozynski, P. P.; Kulinowski, P.; Mlynarczyk, A.; Stanisz, G. J. Foundation review: MRI as a tool for evaluation of oral controlled release dosage forms. Drug Discovery Today 2012, 17, 110-123. DOI: 10.1016/j.drudis.2011.10.026.

6. Charest, K.; Mak-Jurkauskas, M. L.; Cinicola, D.; Clausen, A. M. Fused deposition modeling provides solution for magnetic resonance imaging of solid dosage form by advancing design quickly from prototype to final product. J. Lab. Autom. 2013, 18, 63-68. DOI: 10.1177/2211068212458264.

7. Jambhekar, S. S.; Breen, P. J. Drug dissolution: significance of physicochemical properties and physiological conditions. Drug Discovery Today 2013, 18, 1173-1184. DOI: 10.1016/j. drudis.2013.08.013.

8. Puncochova, K.; Ewing, A. V.; Gajdosova, M.; Sarvasova, N.; Kazarian, S. G.; Beranek, J.; Stepanek, F. Identifying the mechanisms of drug release from amorphous solid dispersions 
using MRI and ATR-FTIR spectroscopic imaging. Int. J. Pharm. 2015, 483, 256-267. DOI: 10.1016/j.ijpharm.2015.02.035.

9. Kulinowski, P.; Hudy, W.; Mendyk, A.; Juszczyk, E.; Weglarz, W. P.; Jachowicz, R.; Dorozynski, P. The relationship between the evolution of an internal structure and drug dissolution from controlled-release matrix tablets. AAPS PharmSciTech 2016, 17, 735-742. DOI: 10.1208/s12249-015-0402-1.

10. Kulinowski, P.; Woyna-Orlewicz, K.; Obral, J.; Rappen, G.; M.; Haznar-Garbacz, D.; Weglarz, W. P.; Jachowicz, R.; Wyszogrodzka, G.; Klaja, J.; Dorozynski, P. P. Multimodal approach to characterization of hydrophilic matrices manufactured by wet and dry granulation or direct compression methods. Int. J. Pharm. 2016, 499, 263-270. DOI: 10.1016/j.jpharm.2015.12.067.

11. Arganda-Carreras, I.; Kaynig, V.; Rueden, C.; Eliceiri K. W.; Schindelin J.; Cardona A.; Seung H. S, Trainable Weka Segmentation: a machine learning tool for microscopy pixel classification. Bioinformatics 2017, 33, 2424-2426. DOI: 10.1093/bioinformatics/btx180.

12. Schindelin, J.; Arganda-Carreras, I.; Frise, E.; et al., Fiji: an opensource platform for biological-image analysis, Nature methods 2012, 9(7), 676-682. DOI: 10.1038/nmeth.2019

13. Lagerlof, F.; Dawes, C. The volume of saliva in the mouth before and after swallowing. J. Dental Res. 1984, 63, 618-621. DOI: 10.1177/00220345840630050201.
14. Szabo, B.; Kallai, N.; Toth, G.; Hetenyi, G.; Zelko, R., Drug release profiles and microstructural characterization of cast and freeze dried vitamin B-12 buccal films by positron annihilation lifetime spectroscopy. J. Pharm. Biomed. Anal. 2014, 89, 83-87. DOI: 10.1016/j.jpba.2013.10.031.

15. Onishi, H.; Yumoto, K.; Sakata, O. Preparation and evaluation of ritodrine buccal tablets for rational therapeutic use. Int. J. Pharm. 2014, 468, 207-213. DOI: 10.1016/j.ijpharm.2014.04.009.

16. Dorozynski, P.; Jamroz, W.; Niwinski, K.; Kurek, M.; Weglarz, W. P.; Jachowicz, R.; Kulinowski, P. Novel method for screening of enteric film coatings properties with magnetic resonance imaging. Int. J. Pharm. 2013, 456, 569-571. DOI: 10.1016/j. ijpharm.2013.08.004.

17. Kulinowski, P.; Woyna-Orlewicz, K.; Rappen, G.-M.; HaznarGarbacz, D.; Weglarz, W. P.; Dorozynski, P. P., An understanding of modified release matrix tablets behavior during drug dissolution as the key for prediction of pharmaceutical product performance - case study of multimodal characterization of quetiapine fumarate tablets Int. J. Pharm. 2015, 484, 235-245. DOI: 10.1016/j.ijpharm.2015.02.040. 